\title{
Remote-Plasma-Assisted Deposition of Pentacene Layer Using Atomic-Hydrogen
}

\author{
Satoshi Yamauchi*, Takatoshi Minakuchi, Miyuki Onodera \\ Department of Biomolecular Functional Engineering, Ibaraki University, Hitachi, Japan. \\ Email: *ysatoshi@mx.ibaraki.ac.jp
}

Received October $22^{\text {nd }}, 2013$; revised November $22^{\text {nd }}$, 2013; accepted November $29^{\text {th }}, 2013$

Copyright (C) 2014 Satoshi Yamauchi et al. This is an open access article distributed under the Creative Commons Attribution License, which permits unrestricted use, distribution, and reproduction in any medium, provided the original work is properly cited. In accordance of the Creative Commons Attribution License all Copyrights @ 2014 are reserved for SCIRP and the owner of the intellectual property Satoshi Yamauchi et al. All Copyright (C) 2014 are guarded by law and by SCIRP as a guardian.

\begin{abstract}
Pentacene thin layers were deposited on $\mathrm{Si}$ with the native oxide at $80^{\circ} \mathrm{C}$ by remote-plasma-assisted deposition (RPAD) using hydrogen-plasma cell to supply atomic hydrogen radicals. The deposition rate was increased by RPAD comparing to that by non-excited hydrogen gas supply whereas thermal evaporation rate of pentacene from crucible was same in the both process. DFM and XRD studies showed the grain laterally grew in the thin film phase with the size above $10 \mu \mathrm{m}$ by RPAD. First-principles molecular orbital calculations suggested pentacene is evaporated from crucible as the trimer or larger cluster but atomic hydrogen penetrated into the cluster enhances cracking of pentacene clusters to the monomer.
\end{abstract}

\section{KEYWORDS}

\section{Remote-Plasma-Assisted Deposition; Pentacene; Hydrogen Plasma; Atomic Hydrogen}

\section{Introduction}

Pentacene is a candidate for organic semiconductors to fabricate organic thin film transistors (O-TFTs) aiming at mechanically flexible and weight reduced devices [1,2] because of the relatively high carrier mobility [3] (1.5 $\mathrm{cm}^{2} / \mathrm{Vs}$ ) close to amorphous $\mathrm{Si}$. In general, pentacene thin films are grown by vacuum evaporation using highpurity pentacene precursor. In the concept of carrier scattering at the grain boundaries, it is considered that the mobility is increased with the grain size. Surface flatness of the channel layer is also taken into account for the device applications to apply uniform electric field. In addition, vacuum evaporated pentacene layer consists of two crystallographic-phases (bulk phase and thin film phase) $[2,4,5]$, which is unfavorable for the device operations because of increasing sample-to-sample variation and decreasing carrier mobility. Previously, Bouchoms et al. studied formation of the two crystallographic phase for the growth condition and the thickness [5]. They indicated pentacene is crystallized into the thin-film phase

\footnotetext{
${ }^{*}$ Corresponding author.
}

consisting of terrace-like structures but the bulk-phase consisting of lamellar-like structures is increased with the growth temperature and the thickness. In contrast, Zhu and Varahramyan demonstrated pentacene deposition on modified $\mathrm{SiO}_{2}$ surface by self-assembled monolayer using poly dimethyldiallylammonium chloride [6]. They suggested pentacene with uniform grain size grows on the modified surface comparing to that on $\mathrm{SiO}_{2}$, which results in improvement of FET device performance such as increase of the effective carrier mobility and the On/Off ratio. On the other, Yokoyama et al. demonstrated hydrogen-gas induced evaporation deposition of pentacene layer with devising the apparatus configuration and optimizing the growth condition, and indicated the grain size is significantly increased with preventing the surface roughness and the notably improved FET performance [7]. The interesting result is concluded that enhanced migration of pentacene precursor on hydrogen-terminated $\mathrm{SiO}_{2}$ results in the improved growth behavior on the grain size and the surface morphology.

In this paper, we demonstrate remote-plasma-assisted deposition (RPAD) of pentacene using hydrogen-gas plasma to improve the crystallographic feature compar- 
ing to that by non-excited hydrogen gas induced vacuum evaporation $\left(\mathrm{H}_{2}-\mathrm{VE}\right)$. In addition, the first-principle molecular orbital calculations are used to investigate the evaporation behavior of pentacene and the role of hydrogen for the evaporated pentacene.

\section{Experimental}

\subsection{Pentacene Deposition}

Figure 1 shows an apparatus for remote-plasma-assisted deposition of pentacene layer, in which back pressure of the reactor was under $6 \times 10^{-4} \mathrm{~Pa}$. Commercially available high-purity pentacene powder provided by Kanto Chemical was used for source material, which was thermally evaporated from a quartz crucible. Si with mirror surface was used for the substrate after chemical cleaning by organic solvent and $\mathrm{H}_{2} \mathrm{SO}_{4}+\mathrm{H}_{2} \mathrm{O}_{2}$ solution. Temperature of the substrate and the crucible was monitored by K-type thermo-couple and controlled by PID controllers. High purity hydrogen gas (7N-purity) introduced into a plasma cell was excited by radio-frequency (RF) power at $13.56 \mathrm{MHz}$ though an inductively coupled electrode, where magnetic field of 3000 Gauss at center of the cell was applied by Neodymium magnets, then supplied into the reactor though an orifice. Pressure in the reactor and the plasma-cell was individually monitored by Schulz gage.

\subsection{Evaluation}

Thickness of deposited pentacene layer was checked by surface profiler (DEKTAK150). Surface morphology was observed by atomic force microscope in dynamic mode operation (DFM: SIMADZU SPM-9600). Crystal

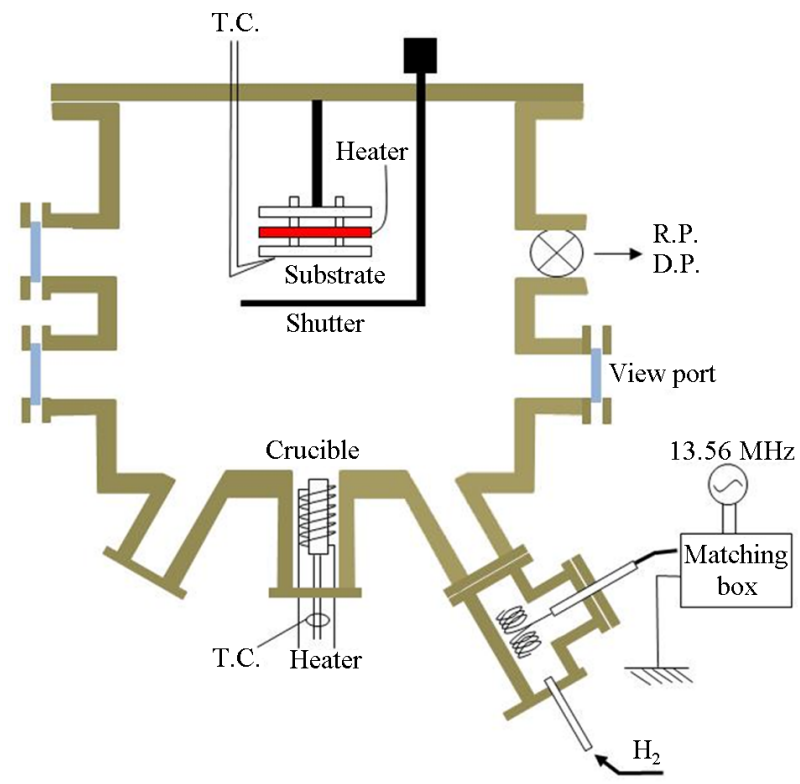

Figure 1. RPAD apparatus for pentacene deposition. lographic phase was determined by $\theta-2 \theta$ X-ray diffraction (RIGAKU: RAD-C) using $\mathrm{Cu}_{\alpha 1}$ and $\mathrm{Cu}_{\alpha 2}$.

Plasma optical emission from the plasma-cell was observed by UV-Vis spectrometer (OCEAN OPTICS: USB-2000) though a quartz-window on the cell.

\subsection{Simulation of Pentacene Precursor}

The GAMESS09 package [8] was used for the first principle molecular orbital calculations of pentacene $\left(\mathrm{C}_{22} \mathrm{H}_{14}\right)$ clusters, in which the Hatree-Fock calculation was used with $6-31++\mathrm{G}(\mathrm{d}, \mathrm{p})$ basis set. The primary set of the bond-angles and the lengths is based on the previously reported data in the bulk-phase crystal [9]. After optimization of the structure for the monomer, dimer, trimer and pentamer by the ab-initio simulation, the total heat of formation was calculated. In the case of hydrogen penetration, the hydrogen atoms or molecules were symmetrically arranged between the pentacene molecules.

\section{Results and Discussions}

\subsection{Hydrogen-Gas Plasma}

Figure 2 shows optical emission spectra of hydrogen plasma generated by $90 \mathrm{~W}$ RF-power in the plasma-cell at the pressure of (a) $5.2 \mathrm{~Pa}$ and (b) $11 \mathrm{~Pa}$, in which the spectra were normalized by the peak intensity of $\mathrm{H}_{\alpha}$. The insets are photographs of the plasmas in plasma-cell. Although a lot of emissions due to hydrogen-molecule were observed with emissions due to excited hydrogen-atom $\left(\mathrm{H}_{\alpha}, \mathrm{H}_{\beta}, \mathrm{H}_{\gamma}\right)$ at $11 \mathrm{~Pa}$, the intensity of emissions due to hydrogen-molecule was notably decreased at 5.2 $\mathrm{Pa}$ comparing to that of $\mathrm{H}_{\alpha}$. Figure 3 shows the emission intensity ratio of $\mathrm{H}_{\alpha} / \mathrm{H}_{2}$ as a function of the cell pressure. The ratio was critically increased with decreasing the pressure below $6 \mathrm{~Pa}$ but decreased under $5 \mathrm{~Pa}$. It is considered that the density of hydrogen-atom is increased with the plasma density which is dependent on the in-

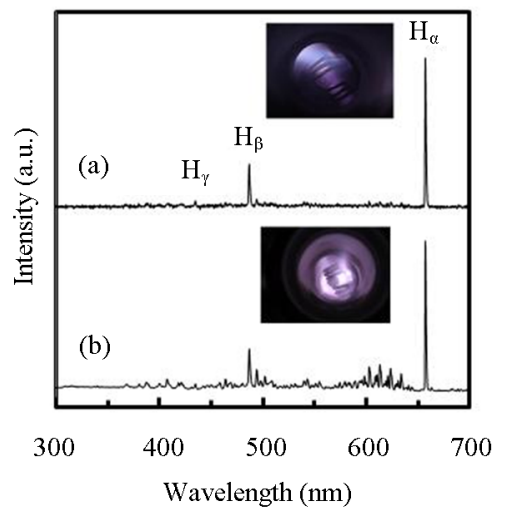

Figure 2. Optical emission spectra from plasma-cell at hydrogen-gas pressure of (a) 5.2 $\mathrm{Pa}$ and (b) 6.4 Pa. The insets are photographs of the hydrogen plasma. 
duced RF-power, the pressure and the cell-configuration. In this work, focusing magnetic field induced by Neodymium magnets was also important to produce the plasma as shown in Figure 2(a). For RPAD of pentacene, the plasma was generated at 5.2 Pa because the ratio was most intense at the pressure. The excited species were introduced into the reactor through an orifice with a hole of $50 \mu \mathrm{m}$-diameter in $100 \mu \mathrm{m}$-thick stainless plate. When the cell-pressure was kept at $5.2 \mathrm{~Pa}$, the reactor pressure was $4.5 \times 10^{-3} \mathrm{~Pa}$. In the process, it was expected that electrons and hydrogen-ions in the plasma were removed or neutralized through the orifice by the plasma-sheath. Then the atomic hydrogen generated in the cell was efficiently supplied on the growth surface because of distance about $20 \mathrm{~cm}$ between the substrate and the orifice in contrast to the mean-free path estimated beyond $2.5 \mathrm{~m}$ in the reactor.

\subsection{Deposition Behavior of Pentacene Layer}

Figure 4 shows (a) thermal evaporation rate of pentacene from crucible as a function of the crucible temperature and (b) the Arrhenius plot, where the reactor pressure was kept at $4.5 \times 10^{-3}$ Pa supplying plasma-cracked hydrogen (closed-circles) and non-excited $\mathrm{H}_{2}$-gas (open-

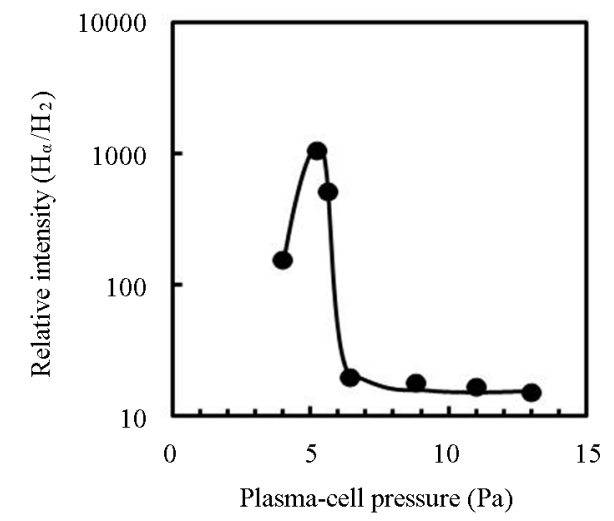

Figure 3. Emission intensity ratio of $\mathbf{H}_{a} / \mathbf{H}_{2}$ in plasma cell as a function of hydrogen gas pressure, where $90 \mathrm{~W}$ RF-power was induced into the cell.
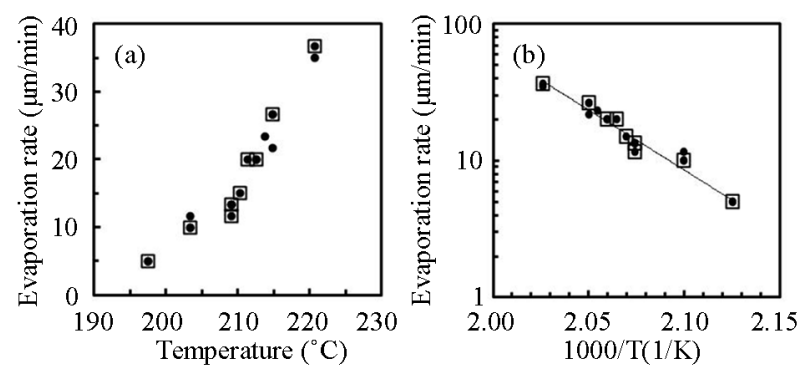

Figure 4. (a) Evaporation rate of pentacene from crucible as a function of the temperature and (b) the Arrhenius plot (closed-circles: plasma-cracked hydrogen supply, opensquares: non-excited $\mathrm{H}_{2}$-gas supply). squares). The evaporation rate was exponentially increased with the crucible temperature and the energy for evaporation was obtained as $40 \mathrm{kcal} / \mathrm{mol}$. Although the plasma-cracked hydrogen did not affect the evaporation, the growth behavior of pentacene was significantly influenced by RPAD using the plasma-cracked hydrogen. Figure 5 shows deposition rate of pentacene by RPAD (open-squares) and $\mathrm{H}_{2}$-VE (closed-circles) at $80^{\circ} \mathrm{C}$ as a function of the evaporation rate from the crucible. The growth rate were uniquely increased with the evaporation rate but higher by RPAD than that by $\mathrm{H}_{2}-\mathrm{VE}$, where the growth rate was obtained by the average thickness of pentacene layer grown for 1 hour. It is noted that any deposits could not be observed for the pentacene evaporation rate below $10 \mu \mathrm{g} / \mathrm{min}$ and $13 \mu \mathrm{g} / \mathrm{min}$ by RPAD and $\mathrm{H}_{2}$-VE respectively, which indicated the precursor could be easily desorbed on the substrate and the growth was limited by the nucleation at the temperature. Figure 6

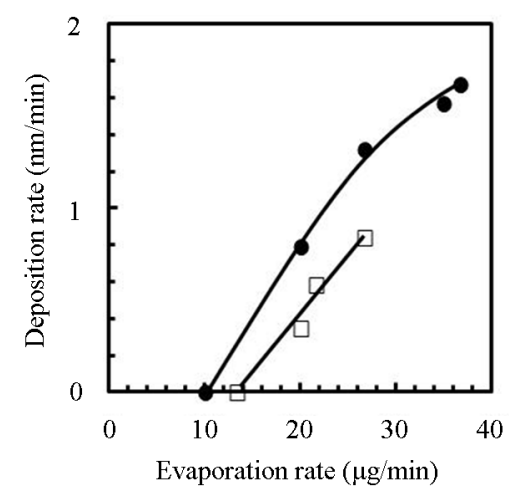

Figure 5. Deposition rate of pentacene by (a) RPAD and (b) $\mathrm{H}_{2}$-gas supply as a function of pentacene evaporation rate from crucible.

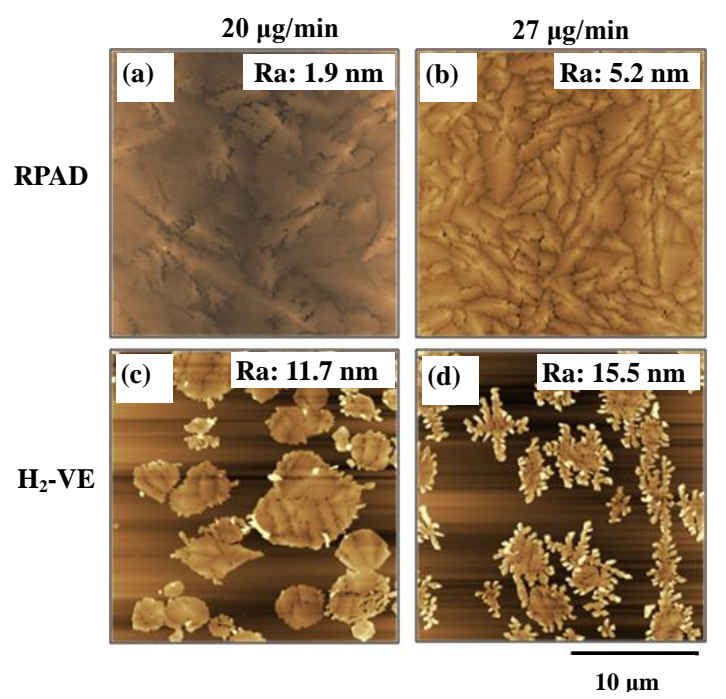

Figure 6. DFM images of pentacene by (a) (b) RPAD and (c) (d) $\mathrm{H}_{2}$-gas supply, where the evaporation rate from crucible was (a) (c) $20 \mu \mathrm{g} / \mathrm{min}$ and (b) (d) $27 \mu \mathrm{g} / \mathrm{min}$. The inset value shows average surface roughness (Ra). 
shows DFM images of the pentacene layers grown by (a) (b) RPAD and (c) (d) $\mathrm{H}_{2}-\mathrm{VE}$, where evaporation rate of pentacene from crucible was kept at (a) (c) $20 \mu \mathrm{g} / \mathrm{min}$ and (b) (d) $27 \mu \mathrm{g} / \mathrm{min}$. Discrete islands dependent on the supply ratio were observed in the pentacene deposited by $\mathrm{H}_{2}$-VE. Grain size and surface roughness of the deposited pentacene was increased and decreased with decreasing the supply rate of precursor respectively. However, the growth feature of discrete and significantly three-dimensional islands suggested that the growth was preferentially performed in the vertical direction on the substrate surface, which probably showed poor migration of precursor on the substrate was resulted in three-dimensional nucleation because the nucleation was originated from adsorption of pentacene to the primary supplied pentacene on the substrate surfaces before the desorption. Additionally, sticking coefficient of supplied pentacene on pentacene nuclei and the island may be higher than that on the substrate surface. In contrast, although the grain size was decreased with increasing supply rate of pentacene, the size was extremely increased with suppressing the surface roughness by RPAD comparing to the results by $\mathrm{H}_{2}$-EV. For example, the largest grain in the sample of Figure 6(a) was beyond $10 \mu \mathrm{m}$ with the step height about $1.6 \mathrm{~nm}$ and the average terrace length about $0.4 \mu \mathrm{m}$. The favorable improvements by RPAD suggested that the two-dimensional nuclei was fabricated and then laterally grown by the migrated molecules on the substrate and the growth surface. The origin of the enhanced migration is discussed in the next section by molecular orbital calculations at the point of view for the supplied pentacene structures.

Figure 7 shows $\theta-2 \theta$ XRD spectra of pentacene grown by (a) RPAD and (b) $\mathrm{H}_{2}-\mathrm{EV}$. The spectrum of Figure 7(a) reveals that the pentacene was dominantly crystallized into the triclinic thin film phase [4] by RPAD because strong and sharp diffraction peak at $5.74^{\circ}$, which corresponds to a lattice spacing of $1.54 \mathrm{~nm}$, was observed with the multiple diffraction peaks for the spacing. In contrast,

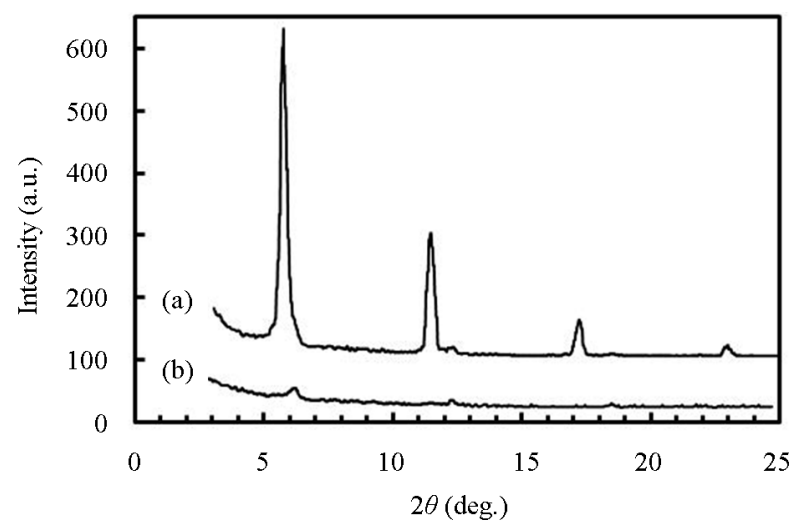

Figure 7. $\theta$-2 $\theta$ XRD spectra of pentacene grown by (a) RPAD and (b) $\mathrm{H}_{2}-\mathrm{EV}$. the diffraction peaks due to the thin film phase were not observed in Figure 7(b) but weak diffraction peak at $6.09^{\circ}$ which corresponds to a lattice spacing of $1.45 \mathrm{~nm}$ was observed with the multiple diffraction peaks. The spectrum indicates that the pentacene was crystallized into the triclinic bulk phase [9] with the degraded crystalline by $\mathrm{H}_{2}-\mathrm{EV}$. Previously, the formation of the two phases in vacuum evaporated pentacene layer was studied for the growth condition such as the growth temperature, the growth rate and the thickness [5]. As a result, it was indicated that the thin film phase is initially formed on $\mathrm{SiO}_{2}$ at low temperatures but the bulk phase is formed on the layer above the critical thickness dependent on the growth temperature. The results in this work show not only such growth condition but also the hydrogen ambient strongly influences to control the crystallized phase with the grain structure.

\subsection{First-Principles Molecular Orbital Calculations of Pentacene Precursor}

First-principles molecular orbital calculations using GAMESS09 package was applied to reveal thermally evaporated pentacene features and contribution of atomic-hydrogen to the evaporated precursor. Figure 8 shows clustering energy of pentacene molecules $(\Delta E)$ calculated by the below relation.

$$
\Delta E=E_{\text {Cluster }}-n E_{\text {Isolate }},
$$

where the $E_{\text {Cluster }}$ and the $E_{\text {Isolation }}$ is the total heat of formation of pentacene cluster with $n(1-5)$ pieces of pentacene molecules and that of isolated molecule respectively. The $\Delta E$ was decreased with the number of pentacene molecules (n) but saturated above 3 molecules, in which the saturated energy about $-350 \mathrm{kcal} / \mathrm{mol}$ was obtained. It is mentioned that the most stabilized structure was parallel configuration of pentacene molecules for the dimer but herringbone structure for the dimer and the pentamer. The result of Figure 8 indicated that the pentacene cluster is more stable than the isolated mole

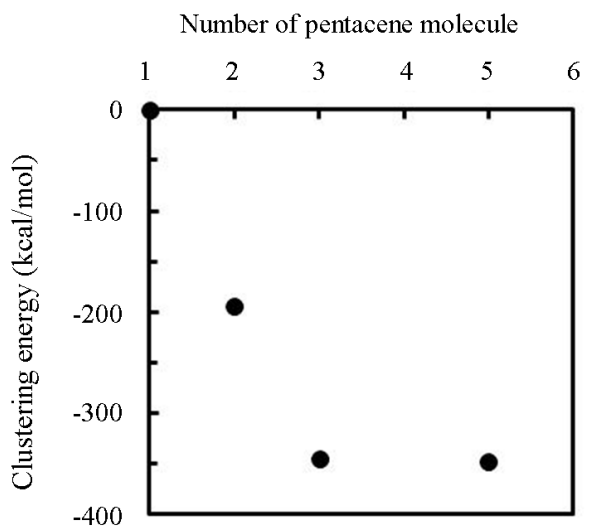

Figure 8. Clustering energy of pentacene molecules. 
cule. Furthermore, the stabilized energy of $350 \mathrm{kcal} / \mathrm{mol}$ for the trimer and the pentamer, which was far large comparing to the evaporation energy of $40 \mathrm{kcal} / \mathrm{mol}$ determined from the result of Figure 4, indicated pentacene was thermally evaporated from crucible as the trimer or larger cluster based on the herringbone structure. As a result, it is considered that migration of the pentacene cluster was significantly limited on the substrate comparing to the monomer. In contrast, when hydrogen was penetrated into the cluster, the system was become to be unstable. Figure 9 shows difference of clustering energy of herringbone pentacene trimer $\left(\Delta E_{\text {Trimer }}\right)$ penetrated hydrogen atoms or molecules for the number of hydrogen atoms or molecules $(\mathrm{m})$ comparing to that of the trimer without hydrogen-penetration, in which the energy was calculated by the below relationship.

$$
\begin{aligned}
& \left.\Delta E_{\text {Trimer }}=E_{\text {Trimer }+m H}-E_{\text {Trimer }}-E_{m \mathrm{H}} \quad \text { (for } \mathrm{H} \text { penetration }\right), \\
& \Delta E_{\text {Trimer }}=E_{\text {Trimer } m \mathrm{H}_{2}}-E_{\text {Trimer }}-E_{m \mathrm{H}_{2}}\left(\text { for } \mathrm{H}_{2} \text { penetration }\right),
\end{aligned}
$$

where the $E_{\text {Trimer }+m \mathrm{H}}\left(E_{\text {Trimer }+m \mathrm{H}_{2}}\right)$, the $E_{\text {Trimer }}$ and the $E_{m \mathrm{H}}$ $\left(E_{m \mathrm{H}_{2}}\right)$ is heat of formation for the pentacene trimer penetrated atomic hydrogen (hydrogen molecules), herringbone pentacene trimer without penetrate-hydrogen and hydrogen atoms (hydrogen molecules) after removal the pentacene molecules, respectively. It is mentioned here that the even number of hydrogen were symmetrically arranged into the pentacene trimer to simplify on the optimization of the cluster. The energy of $\Delta E_{\text {Trimer }}$ is decreased by two hydrogen atoms or two hydrogen molecules comparing to that for pentacene trimer without hydrogen-penetrations, which suggested the trimer is more stable by the hydrogen-penetrations. However, the

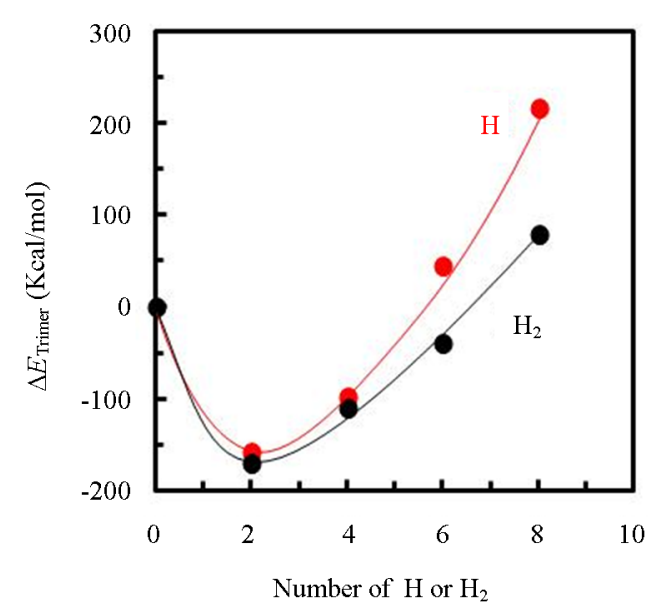

Figure 9. Difference of clustering energy of hydrogen-penetrated pentacene trimer (red-circles: atomic hydrogen penetration, black-circles: hydrogen molecule penetration) comparing to herringbone pentacene trimer without hydrogen penetration for the number of hydrogen.
$\Delta E_{\text {Trimer }}$ was increased with the number of hydrogen for $m \geq 4$, and showed the plus-value by the penetration of hydrogen atoms or molecules with the $m$ above 6 or 8 respectively, which indicated that the trimer was in unstable structures and expected the trimer can be easily separated to the monomer. In addition to the high invasiveness of hydrogen-atom into materials, hydrogenatoms are more efficient for the destabilization of trimer than hydrogen-molecules. As a result, it can be expected that the migration of precursor is enhanced by supply of hydrogen-atoms since the pentacene cluster supplied on the substrate is easily separated to the monomer.

\subsection{Deposition Model}

Figure 10 shows proposed deposition model of pentacene (a) without hydrogen-contribution and (b) with atomic hydrogen supply (RPAD-mode), where the (Sequence 1), (Sequence 2) and (Sequence 3) shows supplying feature pentacene based on the trimer to substrate surface, the nucleation and the island growth respectively. For the deposition without hydrogen-incorporation (Figure 10(a)), the evaporated pentacene trimer supplied on the substrate is adsorbed and then desorbed (Sequence 1). When another trimer is simultaneously supplied on the substrate or adsorbed trimer, the nuclei is formed (Sequence 2) and the island is grown on the surface (Sequence 3). In the process, it is considered that the island is poly-crystallized because the anti-phase is included in
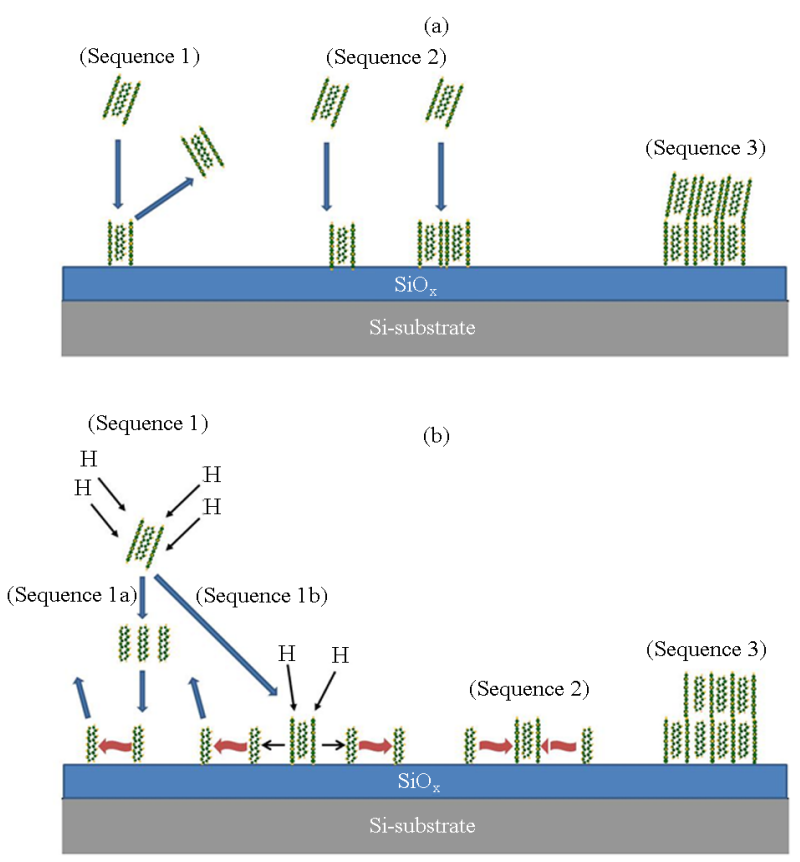

Figure 10. Schematic deposition model of pentacene (a) without hydrogen-contribution and (b) by RPAD, where in the both case "Sequence 1", "Sequence 2" and "Sequence 3 " shows the precursor supply on the surface, the nucleation step and the island growth respectively. 
the nuclei and rearrangement of the precursors is significantly limited. On the other, it is predicted in the case of atomic-hydrogen induced process (Figure 10(b)) that the trimer is cracked to the monomer in vapor phase or on substrate surface and then the monomer is migrated on the surface (Sequence 1). After the migration, the herringbone structure is reconstructed in the nucleation step (Sequence 2). Then the island is grown by the migrated monomer (Sequence 3). It is expected in the process that the island is grown in single domain including the packing.

\section{Conclusion}

Remote-plasma assisted deposition (RPAD) using remotehydrogen gas plasma was applied for pentacene growth comparing the growth by non-excited hydrogen supply. The deposition rate and the grain size of pentacene layer dominantly crystallized into the thin layer phase were increased by the RPAD with significantly suppressing the surface roughness. The first principle molecular orbital calculations revealed pentacene was thermally evaporated as the trimer or more large clusters, however, the cluster can be cracked to the monomer by atomic-hydrogen penetration. Therefore, it was expected that migration of the supplied pentacene was enhanced on the substrate and the growth surface. From the results obtained in this work, it can be concluded that the present RPAD is a sufficient growth process to perform high quality pentacene growth.

\section{REFERENCES}

[1] A. R. Brown, A. Pomp, C. M. Hart and D. M. de Leeuw, "Logic Gates Made from Polymer Transistors and Their Use in Ring Oscillators,” Science, Vol. 270, No. 5238, 1995, pp. 972-974.

http://dx.doi.org/10.1126/science.270.5238.972

[2] Y. Y. Lin, D. J. Gundlach, S. F. Nelson and T. N. Jackson,
"Pentacene-Based Organic Thin-Film Transistors," IEEE Transactions on Electrical Development, Vol. 44, No. 8, 1997, pp. 1325-1331. http://dx.doi.org/10.1109/16.605476

[3] D. J. Gundlach, Y. Y. Lin, T. N. Jackson, S. F. Nelson and D. G. Schlom, "Pentacene Organic Thin-Film Transistors-Molecular Ordering and Mobility," IEEE Electron Device Letters, Vol. 18, No. 3, 1997, pp. 87-89. http://dx.doi.org/10.1109/55.556089

[4] C. D. Dimitrakopoulos, A. R. Brown and A. Pomp, "Molecular Beam Deposited Thin Films of Pentacene for Organic Field Effect Transistor Applications,” Journal of Applied Physics, Vol. 80, No. 4, 1996, pp. 2501-2511. http://dx.doi.org/10.1063/1.363032

[5] I. P. M. Bouchoms, W. A. Schoonveld, J. Vrijmoeth and T. M. Klapwijk, "Morphology Identification of the Thin Film Phases of Vacuum Evaporated Pentacene on SIO Substrates," Synthetic Metals, Vol. 104, No. 3, 1999, pp. 175-178.

[6] M. Zhu and K. Varahramyan, "Performance Improvement of Organic Thin Film Transistors by $\mathrm{SiO}_{2} /$ Pentacene Interface Modification Using an Electrostatically Assembled PDDA Monolayer,” Solid-State Electron, Vol. 50, No. 2, 2006, pp. 129-133. http://dx.doi.org/10.1016/j.sse.2005.10.040

[7] T. Yokoyama, C. B. Park, K. Nagashio, K. Kita and A. Toriumi, "Grain Size Increase and Field-Effect Mobility Enhancement of Pentacene Thin Films Prepared in a Low-Pressure $\mathrm{H}_{2}$ Ambient," Applied Physics Express, Vol. 1, No. 4, 2008, pp. 041801-041803. http://dx.doi.org/10.1143/APEX.1.041801

[8] Gordon Group/GAMESS. http://www.msg.ameslab.gov/gamess/

[9] R. B. Campbell, J. M. Robertson and J. Trotter, “The Crystal Structure of Hexacene, and a Revision of the Crystallographic Data for Tetracene and Pentacene,” Acta Crystallographica, Vol. 15, No. 3, 1962, pp. 289-290. http://dx.doi.org/10.1107/S0365110X62000699 